\title{
Three Kinds of New Mechanism ${ }^{1}$
}

\author{
Arnon Levy \\ The Van Leer Jerusalem Institute \\ To appear in Biology \& Philosophy
}

\begin{abstract}
I distinguish three theses associated with the new mechanistic philosophy concerning causation, explanation and scientific methodology. Advocates of each thesis are identified and relationships among them are outlined. I then look at some recent work on natural selection and mechanisms. There, attention to different kinds of New Mechanism significantly affects of what is at stake.
\end{abstract}

Acknowledgements: Jens Harbecke provided insightful comments on an earlier version of this paper. Written comments from an anonymous referee for this journal, as well as the editor were very helpful, and I am grateful to them.

\section{Introduction}

Mechanisms have been a growing preoccupation of the philosophy of biology over the last two decades. Leading the way is a group of philosophers - prominent names include William Bechtel, Carl Craver, Lindley Darden, Stuart Glennan and Peter Machamer - often referred to collectively as "the new mechanists". Many in the field, I think, view this as a fairly unified bunch, whose members hold a shared a basic picture and advance a common philosophical agenda. But while there are substantial commonalities and overlaps amongst the new mechanists (which may well justify the label), important differences exist too. The goal of this paper is to highlight these differences, as a way of clarifying what is at stake in debates concerning mechanisms.

I will describe three mechanist theses, which concern causation, explanation and the relations among them. ${ }^{2}$ Mechanists have discussed other topics, such as levels and reduction.

\footnotetext{
${ }^{1}$ My title and some terminological choices gesture at Peter Godfrey-Smith's 2001 paper "Three kinds of Adaptationism." The topics are unrelated, but the aims are analogous.
} 
But ideas about causation and explanation form the core, around which the other topics are organized. The three theses are as follows:

1. Causal Mechanism (CM) is the view that causal relations, at least outside the domain of fundamental physical phenomena, exist in virtue of underlying mechanisms. CM rivals other accounts of causation, such as regularity views. It is best seen as a contribution to metaphysics.

2. Explanatory Mechanism (EM) is a thesis about explanatory relevance: it states that to explain a phenomenon, one must cite mechanistic information, i.e. specify underlying parts and their organization. EM contrasts with other general accounts of explanation, such as the Deductive-Nomological model.

3. Strategic Mechanism (SM) concerns the cognitive-epistemic power of mechanistic modelling and related scientific methods. It asserts that certain phenomena are best handled mechanistically. Discussions of SM tend to construe 'mechanism' fairly narrowly, in machine-like terms.

CM, EM and SM aren't always well-marked in the literature, and I suspect this hampers several ongoing debates. In sections 2-4 I look at each view in turn, noting contrasts and pointing to their clearest advocates. Section 5 discusses relationships among the views. In section 6 I illustrate the utility of this taxonomy, by considering what is - or least, what might be - at stake in the debate over whether natural selection is a mechanism. The overall goal of the paper is to promote clarity with regards to discussions of mechanisms. I focus on the ideas, don't engage in much exegesis, and largely refrain from stating my own views.

\section{Causal Mechanism}

Causal Mechanism (CM) is a view about the nature of causal relations. My discussion of CM will be short, because I think it is less central to the philosophy of biology. The key idea in CM is that a causal relation exists between two events (or variables) in virtue of a connecting mechanism. The clearest advocacy of CM is found in Glennan (1996). He defines

\footnotetext{
${ }^{2}$ Nicholson (2011) also recognizes three kinds of Mechanist views. There are some similarities to my discussion, but Nicholson's approach is both more historical and more polemical.
} 
"a mechanism underlying a behavior [as] a complex system which produces that behavior by the interaction of a number of parts according to direct causal laws." (Ibid, 52). Thus, the idea is to account for causal dependencies in terms of interactions among constituents of the system exhibiting the dependence. CM is meant to apply widely - for Glennan, it applies everywhere except fundamental physics. Accordingly he construes key notions in the definition - 'behavior', 'part', 'law' - broadly to apply to a wide range of phenomena, objects and regularities (respectively).

In his original discussion, Glennan offered CM as an answer to Hume's problem, concerning the nature of the "secret connexion" that binds cause and effect. In more recent work, he has also sought to use CM as basis for an account of higher-level properties (2010). Thus, it seems clear that he intends $\mathrm{CM}$ as a contribution to metaphysics.

Glennan stipulates that interactions among parts of a mechanism are "direct". He does not offer an extended discussion of this idea, except stating that if two parts interact directly, then there is no further part intermediate between them (see also his 2005, 446). Other advocates of CM appear to give this notion of directness a different and more central role, marking a distinctive kind of dependency that connects cause and effect (Bogen, 2004; Machamer, 2004; Waskan, 2011). These authors often speak in terms of production, which, at least some of the time, is seen as a distinctive, "actualist" dependence relation, in contrast with counterfactual dependence. ${ }^{3}$

Woodward $(2004,2011)$ has argued that production-based views of causation cannot adequately account for the role of causal notions in science, and for the kinds of evidence that causal claims are sensitive to. Others have suggested that production isn't an alternative to counterfactual dependence, but another and equally valid causal concept (Hall, 2004). This is not the place to delve into these issues. My aim is mainly to highlight that CM is a view about the nature of causal relations.

Apart from the authors just mentioned, I do not know of other mechanists who have expressed support for $\mathrm{CM} .{ }^{4}$ Indeed, at least one prominent mechanist, Carl Craver, has argued against CM, instead adopting Woodward's counterfactual view of causation. This

\footnotetext{
${ }^{3}$ Glennan, it should be noted, does not share this hostility towards counterfactuals. Indeed, over time he has incorporated into his account some central elements of Woodward's (2003) theory.

${ }^{4}$ Machamer, Darden and Craver (2000) uses the language of "production", but in a largely noncommittal way. In a short subsection (3.1) they hint at a deflationary attitude. In any event, as I explain in more detail below, the goal of MDC's paper isn't to give an account of causation.
} 
situation is not surprising. Craver's concerns lie mainly in giving a mechanistic account of explanation. As we shall see, one may pursue that project independently of commitments regarding the metaphysics of causation.

\section{Explanatory Mechanism}

I now turn to Explanatory Mechanism (EM), which is a view about scientific explanation. EM also relies on a notion of mechanism, and in its main advocates one finds characterizations of this notion that are broadly similar to the one cited from Glennan, e.g.:

"Mechanisms are entities and activities organized such that they are productive of regular changes from start or set-up to finish or termination conditions." (Machamer, Darden and Craver, 2000, 3).

"A mechanism is a structure performing a function in virtue of its component parts, component operations, and their organization. The orchestrated functioning of the mechanism is responsible for one or more phenomena." (Bechtel 2006, 26).

For these authors too, mechanisms are complex systems of causally interacting parts that underlie a phenomenon of interest. But these characterizations aren't offered as part of an account of causation. Rather, they form the basis of a view about explanatory relevance. That is to say: they are specifications of the kind of information that successful scientific explanations ought to contain. ${ }^{5}$ According to EM, explanatory relevance has two main aspects: components and organization. A mechanism is a causal structure that does something - it produces a "regular change" (MCD), or "performs a function" (Bechtel). This occurs in virtue of the mechanism's constituents (the underlying "entities" or “component parts"), and how they are situated relative each other, causally and spatiotemporally. Thus, EM tells a two-part story about what kinds of facts are pertinent to understanding: there are the structure and causal powers of components, and there are their interrelations.

Most advocates of EM have remained fairly coy about some of the key notions here. They give examples, but they do not provide explicit accounts of what a part is, how to

\footnotetext{
${ }^{5}$ This is not to say that Glennan does not believe in EM. Indeed he develops a version of it himself (Glennan, 2005). But as I read Glennan, the first order of business for him is providing a mechanistic account of causation. I return to this in section 5 .
} 
construe organization, and which criteria pick out, among possible parts and organizational features, those that are explanatorily relevant. An exception is Craver, who devotes considerable attention to these questions (2007. Ch. 4). Craver discusses criteria for parthood, e.g. that a part must have a stable set of properties, that it must be robustly detectable and that it should be possible to intervene on it. He also provides an account of constitutive relevance, i.e. of which parts are those that matter. This is the "mutual manipulability" account, according to which relevant parts are those that one can intervene upon - sensu Woodward (2003) - to effect a change on the mechanism as a whole, and vice versa. It is not obvious whether other EMers accept these ideas. But it is clear that Craver's efforts are directed at the same end, namely causal-explanatory relevance: specifying which aspects of the causal structure of the world are those that a good explanation must cite.

In illustrating their view EMers sometimes appeal to engineering-like examples (e.g. a mouse trap, in Craver and Bechtel, 2005). More commonly, they point to cases form the proximal parts of biology, such as molecular and cellular biology, genetics and neurophysiology. MDC, for instance, cite the example synaptic transmission. A textbook account of this phenomenon proceeds by, first, describing the relevant parts of the neuron: the membrane and calcium channels embedded within it, calcium-sensitive enzyme cascades within the cell, vesicles loaded with neurotransmitter etc. One then specifies how these are related in the generation of the phenomenon: a change in membrane voltage opens channels that let calcium into the cell; calcium triggers a chain of enzymatic reactions that result in vesicles fusing with the cell membrane and releasing neurotransmitter into the synaptic cleft, etc. Such accounts are often accompanied by diagrams (e.g. Figure 1). 


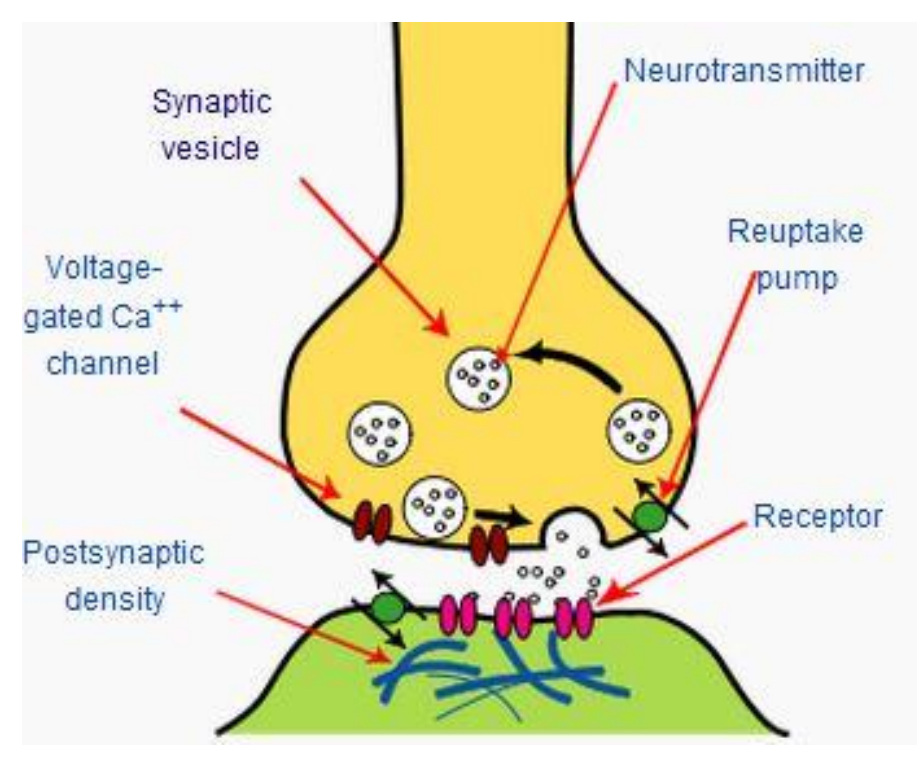

Figure 1. A chemical synapse and some of its elements [Source: Wikipedia]

This example, and others used by EMers, points towards mechanisms with a relatively concrete geometrico-mechanical flavor. Parts have shapes and sizes, interactions are local and often have a push-pull character, and organization consists largely in their layout in space and time. But it seems that most advocates of EM would not want to restrict their view to such machine-like systems. The picture is also meant to cover systems with a more diffuse character, where parts are not so well-defined, and interactions are less regimented in space and time. However, how far the picture is meant to (and can) extend is often unclear. I return to this point later - in the next section, and also in connection with natural selection.

In terms of basic big-picture views of scientific explanation, EM is often contrasted with older non-causal accounts, especially the Deductive-Nomological (D-N) account due to Hempel (1965). The D-N account treated explanation as a matter of logical derivation of the phenomenon to be explained from premises that include at least one law of nature. In EM there is no significant role for either laws or logic. Some EMers highlight the representational means by which mechanistic information is presented, especially graphs and charts. But EM does not take explanations to have a canonical form, nor does it suppose some definite logical relationship between explanans and explanandum. Similarly with respect to the nomological part: mechanisms are seen as exhibiting regular behavior, and advocates may acknowledge that generality is an explanatory virtue. But that is different from saying that explanation is a matter of citing regularities. The D-N account took explanation to consist in subsuming a 
phenomenon under a law - showing that it fits into a lawful pattern. Mechanists view explanation as a matter of showing how a phenomenon depends on the organized interactions among parts.

These last few points mark off EM from D-N explanation, though not from more recent causal views of explanation. Mechanists have been less explicit on this count, so it is harder to make definite claims here. But two aspects of discussions of EM suggest at least a shift in focus, relative to other causal accounts of explanation. First and most importantly, EM is typically concerned with constitutive rather than etiological explanation. That is to say, it is focused on explanations that appeal to underlying structure, rather than to causal history looking down, rather than sideways. This stands in contrast to a lot of work in the causal tradition, which starts with etiology, and often spends fairly little time on constitutive explanation (e.g. Lewis, 1986; Strevens, 2008; Woodward, 2003).

A related shift of emphasis is that EM deals almost exclusively with regularity explanation. Early advocates of the causal approach inherited from the logical positivists a focus on event explanation, and developed accounts that were tailored to fit examples such as explaining why a particular person contracted paresis or why a certain star has collapsed (Lewis, 1986; Salmon, 1984). They largely ignored the explanation of recurrent phenomena. The tendency to treat event explanation as primary still exists, even when more attention is devoted to regularities (Strevens, 2008). EM seems to take a regularity-first approach, looking to account primarily, indeed almost exclusively, for explanations of recurrent phenomena. ${ }^{6}$

To a significant extent, central aspects of EM have to do with a common argumentative strategy of paying close attention to scientific practice, primarily in biology. Other accounts of explanation, especially in the causal tradition, also aim to take account of, and rationally reconstruct, criteria that guide scientists. But Mechanists rely more heavily on detailed casestudies, and place little confidence in informal judgments concerning thought experiments or toy examples. In particular, as noted before, EMers have devoted most of their attention to proximal-physiological parts of the life sciences. In these areas constitutive accounts of recurrent phenomena are ubiquitous.

These points naturally give rise to questions about scope. Some philosophical work on explanation, both in the $\mathrm{D}-\mathrm{N}$ framework and in the more recent causal approach, clearly

\footnotetext{
${ }^{6}$ There is no tension between this point and the earlier claim that EM shuns law-based explanation. The former has to do with the explanans; the latter concerns explananda.
} 
aspires to offer a general account of scientific explanation. EMers sometimes express their view in ways that suggests a similar ambition. ${ }^{7}$ But they typically hedge, and some authors appear to restrict their claims to specific areas of science, like biology (Bechtel and Abrahamsen, 2005) or even subfields thereof (Craver, 2007). To some extent this can be attributed to the argumentative strategy of relying on case-studies, and to a desire not to overextrapolate. It is also connected with the narrower understanding of the notion of mechanism that plays a role in Strategic Mechanism (SM), which I discuss next.

\section{Strategic Mechanism}

So far we have considered CM, a view on causation; and EM, which concerns explanatory relevance. The third view I will look at is Strategic Mechanism (SM), which articulates a way of doing science, a framework for representing and reasoning about complex systems. My use of the term 'strategic' is meant to convey that SM sees mechanistic methods as having particular cognitive and epistemic features, and as a distinctive way of approaching phenomena in certain domains. It outlines a strategy. Many advocates of EM also appear to hold SM, but the difference between these ideas isn't well-demarcated and I believe this generates considerable confusion. Matters of scope, which were alluded to before, play a bigger role in the discussion of SM. The distinctive content of this view depends largely on a certain understanding of what it means to treat a system mechanistically, which draws on a fairly close analogy to machines. The particular forms of representation and analysis highlighted by advocates of SM make most sense for such systems.

The clearest advocacy of SM is found in Bechtel and Richardson's 1993 book "Discovering Complexity". The bulk of it is devoted to two sorts of mechanistic methods: decomposition, i.e. the breakdown of a system into parts; and localization, i.e. the assignment of distinctive causal roles to parts. They acknowledge that these methods, while powerful in some cases, may fail when the conditions aren't right: for instance when components are spread out or otherwise difficult to isolate, or when there isn't enough independence between their roles. Manmade machines like clocks and engines are clear cases where isolatable

\footnotetext{
${ }^{7}$ For instance: "In many fields of science what is taken to be a satisfactory explanation requires providing a description of a mechanism." (MDC, 2000,1). And: "Whether it is geology or molecular biology, biochemistry or cosmology, what is taken to be a causal explanation often consists of the description of a mechanism." (Tabery, 2004, 1). And also: "In many areas of science, explanations are said to be adequate to the extent, and only to the extent, that they describe the causal mechanisms that maintain, produce, or underlie the phenomenon to be explained, the explanandum phenomenon." (Kaplan and Craver, 2011, 601)
} 
components play distinct parts. Bechtel and Richardson state explicitly that they have this analogy in mind (ibid, 17). The analogy readily applies in some cases, e.g. to so-called molecular machines, large enzymatic complexes such as ATPase or the flagellum. But it seems likely (and it is part of Bechtel and Richardson's view) that it can be extended to more diffuse systems, like metabolic pathways and regulatory networks. However, it is also part of SM as I understand it, and as developed by Bechtel and Richardson, that not all biological systems have a machine-like character. The motivation for SM comes in part from assumptions about nature, and biology in particular - namely that important biological systems are sufficiently machine-like - but it also rests on views about the cognitive makeup of human investigators: broadly speaking, that we find piecemeal, relatively concrete, visualizable representation and analysis epistemically congenial.

Thus SM's distinctive content seems to rest on a significant restriction of scope: it is a view about the epistemic utility of a certain class of cognitive methods (roughly associated with decomposition and localization) with respect to a particular set of phenomena (roughly those that are machine-like in their mode of organization). It would seem, from the case studies they look at, that Bechtel and Richardson view decomposition and localization as especially powerful in proximal biology (biochemistry, cell biology and neurophysiology are key areas they discuss). These, as I have noted above, are the areas most frequently drawn on in the literature on mechanisms, and I it is one reason why EM and SM are often not clearly distinguished. Matters are also obscured by the fact that SMers (as well as EMers) tend not to elaborate on contrast cases, in which Mechanism does not apply so well.

Of particular significance is the lack of discussion of statistical and populational phenomena and the disciplines devoted to their study. Thus, there is little mention of processes like diffusion and other statistical mechanical processes, even as they apply to living beings. Nor is there discussion of population genetics, ecology and other macrobiological populational disciplines. Intuitively, in these areas methods such as decomposition and localization are expected to play a lesser role. Modelling and discovery is not as concerned with distinctions among parts, or in charting locations and spatiotemporal layout. Bechtel and Richardson come closest to discussing such cases when they allude to Wimsatt's (1986) notion of aggregativity - cases in which overall behavior is a cumulative, "summary" function of the behavior of a large collection of parts. They note that in truly aggregative systems, decomposition and localization are unlikely to yield understanding. And they add that "few interesting dynamic systems are truly aggregative." (ibid, 25. Craver, $\S 4.7$ makes 
similar remarks). This may appear to be a statement about the empirical insignificance of aggregative processes. But read in context I think it is more plausibly understood as a statement about the scope of SM, i.e. the range of phenomena to which the methods of decomposition and localization can be fruitfully applied. Indeed they go on to discuss systems that vary in their degree of aggregativity, indicating that the mechanistic strategy is expected to yield better results the further away one is from a "true aggregates."

As noted, I think Bechtel and Richardson (1993) offer the clearest formulation of SM, as a distinct project within the Mechanist literature. But the ideas play a significant role in other authors too. For instance, Darden discusses at length strategies for mechanistic explanation and discovery (2006, especially chapters 3, co-authored with Craver, and 10). Though officially adhering to the definition of 'mechanism' from MDC (2000), cited above, much of what she says seems to be most relevant to a narrower range of cases. The strategy of schema instantiation is one example. Mechanism schemata are descriptions that "specify roles, black boxes, at varying degrees of abstraction with more or less detail specified. The schema terms can then be filled with the mechanism's entities and activities as they are hypothesized and discovered." (Darden, 2006, 86-87). If the explanatory procedure proceeds by assigning different roles to different parts, then it would seem out of place where parts do not have distinct roles, as is the case in many aggregative or populational phenomena. Darden also discusses the method of probing a mechanism at different points along its progression, which she labels forward/backward chaining. "Looking forward," she says, "each stage must give rise to, allow, drive, or make the next. Conversely, looking back, each stage must have been produced, driven, or allowed by the previous stage(s)." (Ibid, 68). Here, too, there is an underlying picture of the objects of study as internally regimented, in particular as akin to a "production line" (Darden discusses the example protein synthesis). Like schema instantiation, forward/backward chaining appears less at home in cases where the underlying structure is more diffuse in character, as it is in statistical and populational phenomena. So, an important theme in Darden's work is the utility of certain investigative methods, which seem most appropriate in particular empirical domains.

Let me briefly mention another place where a commitment to Strategic, rather than other forms of Mechanism is visible - the discussion of graphical models (e.g. Bechtel and Abrahamsen, 2005; Craver and Bechtel, 2006). Bechtel and Abrahamsen, for instance, state that "[a] diagram provides a vehicle for keeping in mind the complex interactions among operations" within a mechanism (Ibid, 427). A key reason, they suggest, is that "spatial 
layout and organization is often critical to the operation of a mechanism. As in a factory, different operations occur at different locations. Sometimes this serves to keep operations separate from one another and sometimes it serves to place operations in association with one another. These spatial relationships can be readily shown in a diagram." (Ibid, 428). Bechtel and Abrahamsen are remarking on a cognitive strategy, in this case a method for representing explanatory information. Their comments suggest that they think of this method as best directed towards a particular class of objects - those in which "spatial layout and organization" are "critical".

In sum, SM is the idea that there is a characteristically mechanistic style of doing science, which involves particular methods of representation, reasoning and understanding. SM's distinctive content stems from a relatively narrow, machine-like, construal of the notion of mechanism. The methods typically highlighted by SMers are ones that can be expected to yield progress with respect to such systems - where parts play distinct roles, and delicate organizational features make a difference.

\section{Relations between Causal, Explanatory and Strategic Mechanism}

Having laid out the different Mechanist theses, I now want to discuss some relations among them. I will look at connections between CM and EM and between EM and SM. (The connections between CM and SM are weaker and less direct, and I will set them aside here). The emphasis is on significant combinations of views, and not on which combinations are held by which philosophers.

5.1. Causal and Explanatory Mechanism. It seems clear that CM and EM are logically distinct theses. One may hold that causal relations exist in virtue of underlying mechanisms (CM) while denying that only, some or even any information about mechanisms is explanatorily relevant. Vice versa too: a person may believe that to understand a phenomenon one must look under the hood and discern its underlying structure (EM), while denying that such structures are definitive of causation.

That said, a CMer is likely to also be an EMer. That is to say: if one holds that causation is a matter underlying mechanisms, then one is likely to take a mechanistic view of explanatory relevance. There are at least two reasons for this. First of all, a causal view of explanation (specifically, of explanatory relevance) is independently compelling (Salmon, 1989; Strevens, 2006; Woodward, 2009). If one views causation along mechanistic lines, then one is likely to think that mechanistic information is at least part of the story about 
explanatory relevance. The second reason is more internal to CM: intuitions about the explanatory value of looking "under the hood" are part of the motivation for CM (Glennan, 2010). It is partly because of the role such information plays in explanation that it seems, to some, a good way to approach causation.

Movement in the other direction looks less compelling: as such, an adherence to EM need not motivate CM. EM is a view about the kind of information that is pertinent to explanation, information regarding parts and their organized interactions. Such a view can be joined to any number of views about what constitutes causal relations, and part-part interactions in particular. Put differently, looking at interactions amongst parts within a complex system does not lend special support to, say, a mechanistic take on causation over a counterfactual or regularity view. Thus, an EMer might hold CM, but that is likely to be on independent grounds.

These remarks comport well with the views of at least two prominent mechanists. Stuart Glennan, as discussed in section 2, advocates CM. In large part because of this, he also espouses EM (2002). For Glennan, causes boil down to mechanisms and explanation is causal. Therefore, explanations are mechanistic. No doubt this is a coherent package. Another package is found in Craver (2007). He rejects CM for reasons that are largely metaphysical, and at any rate independent of his explanatory mechanist outlook. Instead he favors the counterfactual approach to causation of Woodward (2003). Indeed, as noted above, he makes use of the interventionist framework in developing part of his brand of EM. Thus, Craver rejects $\mathrm{CM}$ and in favor of a different view of causation.

5.2. Explanatory and Strategic Mechanism. The relationship between EM and SM is more complicated, and requires attending to a background issue: - the difference between the ontic and the epistemic conceptions of explanation. These terms are due to Wesley Salmon (1989), and they are widely used to designate different frameworks for philosophical theorizing about explanation. Important details vary among authors but the basic idea is this. To hold an ontic conception is to view explanation as primarily a matter of explanatory facts, in particular causal facts. Accounting for these facts, in objective terms, is seen as the main task of a philosophical theory of explanation. In contrast, an epistemic conception treats explanation as, first and foremost, a matter of representing the world a certain way. The primary task of a philosophical theory of explanation, on this conception, is to account for explanatory representations - a more subjective, or human-relative matter (for more on the ontic conception see: Levy, forthcoming). Some Mechanists explicitly adhere to the ontic 
conception, while others express support for the epistemic conception. Not coincidentally, the latter tend to advocate SM, the former not so much. Let me explain.

As I have characterized it, EM is a view about explanatory relevance. It states what kind of facts in the world a successful explanation must attend to, namely underlying parts and interactions. Both an onticist and an epistemicist about explanation can accept this view. But the former focuses on giving an account of the relevant facts, whereas for the latter the action is in accounting for how science represents and reasons about said facts. This has the result that an epistemicist about explanation who holds EM will tend to focus on how mechanistic information is represented in explanation. This is indeed what we see, for instance, in Bechtel and Abrahamsen, who explicitly endorse the epistemic conception (2005). They give a short characterization of mechanisms "as found in nature" (Ibid, 423), but then devote most of their discussion to mechanistic models and other representational devices. Obviously, this type of discussion will shade into SM, insofar as it also is concerned with methods of representation, reasoning and investigation. SM's distinctive content, as discussed above, comes from its emphasis on machine-like systems and the scientific strategies they are amenable to. But the boundary between more and less machine-like systems isn't sharp, and neither is the distinction between explanatory methods and other aspects of mechanistic science.

A useful contrast to Bechtel and Abrahamsen is Carl Craver, who is an avowed supporter of the ontic conception $(2007,27)$. Accordingly, his book is devoted to an account of the kinds of facts that are pertinent to explanation - causal and constitutive relations, parts and operations and other objective aspects of mechanisms. While Craver does not deny that there is a representational aspect to explanation, he does not see it as the bread and butter of a philosophical account in this area. Craver's adherence to EM does not require that he reject SM. And indeed some of his work is best seen as a contribution to SM (especially papers coauthored with Darden). But for him the two projects are largely distinct. EM has the task of specifying criteria for causal-explanatory relevance. SM delineates strategies of investigation. The strategies may be aimed at getting causal-explanatory information, but for an onticist like Craver, these are different topics. Thus, there is a much looser connection between EM and SM if one views explanation via an ontic lens.

\section{Natural Selection and the New Mechanism}


In this penultimate section I will employ the distinctions drawn so far to look at one recent debate within the mechanisms literature, concerning natural selection. I will not be advancing substantive views: my goal is to clarify what is, or might be, at stake.

The relationship between mechanisms and natural selection is interesting in part because, as noted, the new mechanistic philosophy was developed in the context of proximalphysiological disciplines. Looking at evolution is therefore an opportunity to ask comparative questions about causation and explanation in biology. It is also a chance to think about philosophical methodology, and about the virtues and perils of paying close attention to the practice of a circumscribed portion of science.

I will focus on Skipper and Milstein (2005), although much of what I say is relevant to follow-ups and related papers (e.g. Barros, 2008; Havstad 2011; Pâslaru, 2009). Skipper and Milstein stated aim is to ascertain whether "natural selection - a putative evolutionary mechanism, and a main one at that — can be adequately characterized under the rubric of the "new mechanistic philosophy"." (2005, p. 327). At other points they put the matter in terms of whether the New Mechanism "gets at" or "captures" natural selection.

Their strategy is to construct a schematic representation of natural selection, in the form of an annotated flow chart. They probe the fit of this chart with two of the main characterizations of mechanisms, Glennan's (2002) and MDC's (2000), raising three major concerns. First, under the heading of 'organization', they question whether natural selection involves 'parts' or underlying 'entities', as these are key notions in the relevant definitions of mechanism. Second, under 'productive continuity', they raise doubts about whether the kinds of causal interactions that are countenanced by mechanists (property changes in the case of Glennan; a list of paradigmatic 'activities' in the case of MDC) appropriately characterize natural selection. Finally, they worry about regularity. Both Glennan and MDC require that a mechanism exhibit a measure of regularity (although they have somewhat different attitudes towards the kind and degree of regularity involved). But natural selection is a stochastic process. Milstein and Skipper seem to think that Glennan's account can accommodate the stochasticity of evolution by natural selection better than the MDC account, but they do not see this as a trouble-free issue.

Many of the concerns raised by Skipper and Milstein, as well as the responses to them by subsequent writers, stem from deep issues, concerning the basic constituents of natural selection, their causal efficacy and the relation between processes at the population level and at lower levels. But there is considerable unclarity as to what is at stake in deciding whether 
natural selection is "adequately characterized under the rubric of the "new mechanistic philosophy". We can see this by framing the issue with respect to the three kinds of New Mechanism distinguished earlier.

6.1. Natural Selection and Causal Mechanism. First, consider Causal Mechanism the metaphysical view that mechanisms underwrite causal relations. The key idea in CM is that a system-level causal relation holds in virtue of an underlying structure of interacting parts. As a big-picture view of causation, CM contrasts with regularity views and, for some of its proponenets, with counterfactual accounts. Vis-à-vis such views, the distinctive aspect of $\mathrm{CM}$ is the emphasis on bottom-up dependence of causation at one level on structured lowerlevel interactions. Different formulations of the notion of mechanism then serve as different glosses on what such an underlying structure consists in.

To address $\mathrm{CM}$ in the context of natural selection, one would need to suppose that evolution by natural selection is a causal process. This is a controversial claim (e.g. Matthen and Ariew, 2002; Riesman and Forber, 2005; Walsh, 2010). But if the discussion of natural selection is to have implications for $\mathrm{CM}$ - if it is to be a test case for that view - then something along these lines must be supposed. ${ }^{8}$ However, if one thinks that natural selection is a causal process in virtue of which evolutionary change occurs (as opposed to a statistical epiphenomenon, say), then very likely one thinks of it as a two-tiered affair, with populational changes occurring in virtue of underlying constituents that interact in a structured manner over time. Skipper and Milstein's discussion may then be seen as asking whether existing formulations of mechanism are couched in the appropriate vocabulary. Are the 'parts' and 'interactions', 'entities' and 'activities' countenanced by Mechanists appropriate for "getting at" the constituents of selection? In particular, can populations and the changes they undergo be treated in these terms? Are the organizational features most commonly discussed in New Mechanist texts - spatial layout, temporal sequences etc. - a good match to what we see in evolution? Is the regularity that Mechanists recognize of apiece with the probabilistic character of selection?

As noted above, Skipper and Milstein raise these and related questions, which are undoubtedly important. But it is significant that insofar as CM is concerned, the case of natural selection would seem, at most, to require substantial revisions in formulation. It would not militate against the basic idea of viewing causal relations as underpinned by

\footnotetext{
${ }^{8}$ Skipper and Milstein do indeed speak of natural selection in causal terms throughout. They describe their schematic graphic as "laying bare the causal crux of selection" $(2005,329)$
} 
constituents and their interactions. To repeat, this is because if natural selection is treated as a test case for CM, then it is ipso facto assumed to be a causal process. And if it is seen as a causal process, then it is hard to see how it would not involve some kind underlying structure of interacting elements. Ascertaining whether formulations of mechanism "get at" natural selection might affect important matters of detail with respect to $\mathrm{CM}$, but it is unlikely to motivate one to move from CM to another big-picture view of causation. Thus, in asking whether CM can accommodate the case of natural selection, what is at stake is most probably a reformulation of the notion of mechanism, but not an abandonment of the mechanist view of causation.

6.2. Natural selection and Explanatory Mechanism. The situation is different with respect to Explanatory Mechanism (EM). Recall that EM is the view that explanations ought to provide mechanistic information - i.e. they depict underlying constituents and their organization. Seen in light of EM, the discussion of natural selection targets its role in explanation, not its status as a causal process. As noted earlier, EM was developed with an eye to proximal, especially intra-organismal parts of biology, such as cellular and molecular biology and neurophysiology. The issue here is whether a Mechanist take on explanatory relevance extends beyond those parts of biology, into evolution by natural selection. In other words, vis-à-vis EM, Skipper and Milstein may be seen as raising the following kind of question: in the contexts in which EM has been developed (such as cell biology), information about parts and organization plausibly plays a lead role in explanation. Can the same be said for explanations that appeal to natural selection? ${ }^{9}$

Importantly, one can take a stand on this question independent of one's views on causation. One may well accept that organisms, or other well-defined constituents, play a causal role in evolution by natural selection, but still think that the best explanation of the workings of selection is given in populational terms. Similarly with respect to regularity and stochasticity. One may hold (or at least, see no reason not to hold) that, insofar as the causal realities of natural selection are concerned, all the interactions involved are deterministic and governed by strict fundamental laws. But this is compatible with thinking that evolution by natural selection is best explained probabilistically and/or by appeal to less-than-strict regularities.

\footnotetext{
${ }^{9}$ Skipper and Milstein speak in terms of explanation at several points in the paper, but they never quite say whether they regard explanation-talk as different from causation-talk and if so, how.
} 
An example of someone who holds the latter set of views is Michael Strevens (2008, Chapters 9, 12). His views were not fully developed when Skipper and Milstein's wrote their paper, but from the present vantage point it is instructive to consider them. Strevens argues that in some contexts, among them some cases of natural selection, the best explanation for a deterministic, constitutive phenomenon is probabilistic and populational: it does not operate by attending to parts and underlying organization, but tracks global statistical patterns. I will not recap Strevens' intricate argument here, except noting that his view is based on general considerations about the nature of explanation and probability. It is possible to view the problems Skipper and Milstein raise, problems such as whether natural selection has parts, whether it is regular in the requisite ways etc., as pointing to a similar conclusion, albeit on grounds having to do with evolution in particular. The key point is this: the case of natural selection may, if Skipper and Milstein (and, in another way, Strevens) are right, cause trouble for the key tenets of EM. For it is, potentially at least, a case in which underlying parts and organization aren't explanatorily relevant.

Thus, if explanation is what is at stake, it is possible that the discussion of natural selection motivates a rejection of EM's basic idea, or at least an acknowledgement that it does not apply as well to phenomena like evolution by natural selection.

6.3. Natural Selection and Strategic Mechanism. Finally, the debate over natural selection may concern the viability of the mechanistic strategy. Recall that SM highlights a certain way of doing science, involving decomposition, localization and associated methods. SM points to the special features of machine-like systems that make them amenable to these forms of modelling, representation and discovery. Cast in terms of SM, one might wonder whether natural selection is amenable to mechanistic science, and if so in what ways and to what extent. Here a set of questions concerning methodology and representational practices across different parts of biology arises. ${ }^{10}$

If $\mathrm{SM}$ is at stake, then the issue is likely to turn on which methods are being considered and what kind of questions concerning evolution and natural selection they are directed at. It is possible, however, two distinguish two sorts of attitudes that might be exhibited in such a discussion. One may take the approach that the applicability of mechanistic methods is chiefly a function of the causal structure of natural selection and kindred populational

\footnotetext{
${ }^{10}$ Skipper and Milstein's discussion does not make explicit reference to methods and strategies. But their choice to represent natural selection in graphic form - a relatively unusual attempt - suggests that such issues are in the back of their minds.
} 
processes. This would require comparing the causal structures of natural selection on the one hand, and the kinds of systems which are paradigm targets of mechanistic methods machine-like structures within organisms - on the other hand. Someone who took this line would regard answering the kinds of questions Skipper and Milstein are asking - does natural selection have parts, does it exhibit productive continuity, etc. - as the way to settle questions about SM.

A recent paper by Matthewson and Calcott entertains a different, somewhat less "realist" approach: that one may apply mechanistic thinking to natural selection even if it is not a mechanism. Matthewson and Calcott put the point thus: "What must be addressed is whether or not natural selection can be represented as though it is a mechanism...” $(2011,754)$. In suggesting this, they presuppose that mechanisms are "objects with well-defined and localizable parts which interact in discrete ways" (Ibid, 737). Their suggestion is that one may usefully model a system this way, even if as a matter of fact, it is has a more diffuse, populational character. One might be led to this way of thinking if one holds that the value of mechanistic methods is strongly associated with the cognitive strengths and limitations of scientists, such that representing systems $a$ s if they were mechanistic (in a narrow, machinerelated sense) is a fruitful method because of how it allows one to think and operate, even if its match up with objective features of the target isn't very tight.

One way or another, the debate over natural selection looks different if it is seen as involving SM. It is a debate over the viability of a certain family of methods. One's stance on such issues may, but need not, depend in a direct way one's views about the sorts of systems under investigation.

\section{Conclusion}

I have sought to disentangle three distinct theses within the new mechanistic philosophy - concerning causation, explanatory relevance, and a certain set of scientific methods. Discussions of mechanisms often move unawares between these ideas, or fail to make explicit which one of them is at stake. The case of natural selection - and perhaps populational process more generally - is one place where attending to these issues should significantly affect the framing of such debates. 


\section{REFERENCES}

Barros, D. Benjamin. (2009), Natural Selection as a Mechanism, Philosophy of Science 75 (3): 306-322.

Bechtel, William. (2006), Discovering Cell Mechanisms: the Creation of Modern Cell Biology, Cambridge University Press.

Bechtel, William and Robert C. Richardson (1993/2010) Discovering Complexity:

Decomposition and Localization as Strategies in Scientific Research, Princeton, NJ: University Press.

Bechtel, William and Adele Abrahamsen (2005), Explanation: A Mechanist Alternative, Studies in History and Philosophy of Biological and Biomedical Sciences, 36:421-441.

Bogen, Jim. (2004), Analyzing Causality: The Opposite of Counterfactual is Factual, International Studies in the Philosophy of Science, 18(1): 3-26.

Craver, Carl F. (2006) When Mechanistic Models Explain, Synthese,153: 355-376.

--- (2007). Explaining the Brain: Mechanisms and the Mosaic Unity of Neuroscience, NY: Oxford University Press.

Craver Carl F. and William Bechtel, (2006) Mechanism. In Dubitzky et al. (Eds.), Encyclopedia of systems biology, New York: Springer.

Darden, Lindley. (2006) Reasoning in Biological Discovery, Cambridge: Cambridge University Press.

Glennan, Stuart. (1996) Mechanisms and the Nature of Causation, Erkenntnis, 44: 49-71.

--- (2002) Rethinking Mechanistic Explanation, Philosophy of Science, 69: S342-S353.

--- (2005), Modeling Mechanisms, Studies in History and Philosophy of Science Part C, 36 (2):443-464.

Godfrey-Smith, Peter. (2001), Three Kinds of Adaptationism, in Orzack, Steven H. and Elliott Sober (Eds.), Adaptationism and Optimality, Cambridge University Press.

Hall, Ned. (2004), Two Concepts of Causation, in Collins, J., Hall, N. \& L.A. Paul, Causation and Counterfactuals, Cambridge, MA: MIT Press.

Havstad, Joyce. (2011), Problems for Mechanism \& Natural Selection, Philosophy of Science, 78(3), 512-523.

Hempel, Carl G. (1965), Aspects of Scientific Explanation, New York: Free Press.

Machamer, Peter, Lindley Darden, and Carl F. Craver. (2000). Thinking about Mechanisms, Philosophy of Science, 67: 1-25. 
Matthen, Mohan and André Ariew. (2002), Two Ways of Thinking about Fitness and Natural Selection, Journal of Philosophy, 99 (2):55-83.

Matthewson, John and Brett Calcott. (2011), Mechanistic Models of Population-Level Phenomena, Biology and Philosophy, 26 (5):737-756.

Pâslaru, Viorel. (2009), Ecological Explanation Between Manipulation and Mechanism Description, Philosophy of Science, 76 (5): 821-837.

Reisman, Kenneth and Patrick Forber (2005). Manipulation and the Causes of Evolution. Philosophy of Science, 72 (5):1113-1123.

Salmon, Wesley C. (1989), Four Decades of Scientific Explanation, in Kitcher, Philip and Wesley C. Salmon. (eds.), Scientific Explanation. Minneapolis: University of Minnesota Press.

Skipper, Robert and Roberta Milstein. (2005), Thinking about evolutionary mechanisms: natural selection, Studies in History and Philosophy of Biological and Biomedical Sciences, 36:327-347.

Strevens, Michael. (2008) Depth: An Account of Scientific Explanation, Cambridge, MA: Harvard University Press.

--- (2006). Scientific Explanation. In D. M. Borchert (ed.), Encyclopedia of Philosophy, $2^{\text {nd }}$ edition. Detroit: Macmillan Reference USA.

Tabery, Jim. (2009), Difference Mechanisms: Explaining Variation with Mechanisms, Biology and Philosophy, 24(5): 645-664.

Walsh, Dennis, M. (2010), Not a Sure Thing: Fitness, Probability, and Causation, Philosophy of Science, 77 (2):147-171.

Waskan, Jonathan. (2011), Mechanistic Explanation at the Limit, Synthese, 183(3): 389-408.

Wimsatt, William. (1986), Forms of Aggregativity, in Donagan, Alan, Perovich, Anthony, N. and Michael V. Wedin (eds.), Human Nature and Natural Knowledge, Dordrecht: Reidel.

Woodward, James. (2003), Making Things Happen: a Theory of Causal Explanation, Oxford University Press.

--- (2004), Counterfactuals and Causal Explanation, International Studies in the Philosophy of Science, 18 (1):41-72.

--- (2009), Scientific Explanation. in Edward N. Zalta, The Stanford Encyclopedia of Philosophy, http://plato.stanford.edu/archives/fall2009/entries/scientific-explanation/

--- (2011), Mechanisms Revisited, Synthese, 183(3): 409-427. 